PANDORA'S

BOX

Pandora searches the world literature for evidence, news and other sources o of interest (doesn't shy away from controversy) to bring to the reader. She welcomes comments and suggestions (via ip@rcpsych. ac.uk)

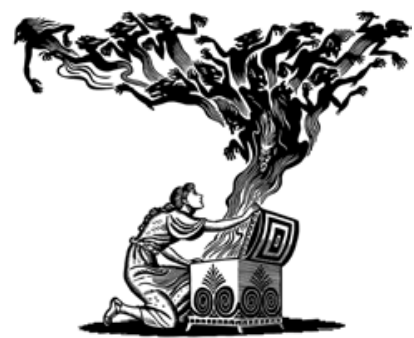

Lewy body dementia - you can diagnose it in no time at all!

J f you have been struggling with the differential 1 diagnosis of dementia you can now rest assured that at least Lewy body dementia should not be a challenge anymore. A recent study claims to make its diagnosis quick and easy through the use of a tool called the Lewy Body Composite Risk Score (LBCRS), which can distinguish Lewy body dementia from other forms of dementia.

It is a simple test that takes no more than a few minutes and consists of ten questions that cover motor symptoms (slowness of movement, balance problems, falls and resting tremor) and non-motor symptoms (sleep, illogical thinking, staring spells, visual hallucinations, acting out dreams and orthostatic hypotension). The answers are 'yes' or 'no' and three or more 'yes' responses suggests that Lewy bodies are the cause of the dementia, according to the author. Simple!

Galvin, J. E. (2015) Improving the clinical detection of Lewy body dementia with the Lewy body composite risk score. Alzheimer's and Dementia: Diagnosis, Assessment and Disease Monitoring, 1 , 316-324.

\section{Is it all to do with the prions?}

re Alzheimer's disease and Parkinson's disease Ain fact prion disorders? A review in Science gathers recent evidence to argue that the scope of prion diseases should be extended beyond Creutzfeldt-Jacob disease (CJD), kuru and scrapie and include other neurodegenerative disorders such as Alzheimer's disease and Parkinson's disease. This could make one cringe but there are good arguments in favour of this view.

Alzheimer's disease is characterised neuropathologically by brain atrophy and the accumulation of extracellular $A \beta$ plaques and intraneuronal neurofibrillary tau lesions.

The term 'prion' (proteinaceous infectious particle), previously known as 'slow virus', was introduced in order to make a distinction between protein pathogens (replicating without nucleic acid) and viruses. Prions were at first thought to be limited to a group of diseases such as CJD in which glycolipid anchored sialoglycoprotein adopts a conformation rich in $\beta$-sheet. However, as similar conformations have been observed for $A \beta$, tau and $\alpha$-synuclein upon assembly, it is argued that the prion concept should also apply to Alzheimer's disease and Parkinson's disease.

An argument against the expansion of the concept is that, unlike CJD and kuru, there is no evidence of transmission between individuals with Alzheimer's disease or Parkinson's disease. However, the author argues, human prion diseases are not easily communicable or contagious; only $1 \%$ of cases of CJD are acquired, while $99 \%$ are sporadic or inherited. In order to confirm this there needs to be evidence that a large increase in the titre of 'infectivity' is observed in assembled $\mathrm{A} \beta$, tau and $\alpha$-synuclein, as occurs after injection of misfolded prion protein in the brain.
The review concludes that trans-cellular propagation of protein pathogens, reminiscent of the spread of viruses, represents an unprecedented concept of disease and claims that it is now known to extend beyond CJD, to include Alzheimer's disease and Parkinson's disease. At present there is no effective treatment for neurodegenerative disorders. Understanding the molecular processes that lead to the formation of inclusions, where they begin to behave like prions, and their routes of transneuronal spread as well as the role of glial cells in the latter may be critical to the development of therapies that can tackle these conditions.

Goedert, M. (2015) Alzheimer's and Parkinson's diseases: the prion concept in relation to assembled $\mathrm{A} \beta$, tau, and $\alpha$-synuclein. Science, 349, doi: $10.1126 /$ science. 1255555 .

\section{Are we overdiagnosing ADHD in children?}

$\mathrm{E}$ leven per cent of children in the USA are diagnosed with attention-deficit hyperactivity disorder (ADHD) and the numbers are rising. The investigators present data from a 2014 nationally representative survey that was sponsored by the Centers for Disease Control and Prevention's National Center for Health Statistics. Households providing data for this report all had children aged $2-15$ years. About $31 \%$ of the children were diagnosed before the age of 6 years and as many as $76 \%$ of the diagnoses were made before the age of 9 years. The ADHD diagnoses were made by paediatricians in $39 \%$ of the children, by other primary care physicians in $14 \%$, by psychologists in $14 \%$, psychiatrists in $18 \%$ and neurologists in $5 \%$, with the remainder of the diagnoses made by other types of providers.

The conclusion was that in the USA, nonpsychiatrists and non-psychologists make most of the diagnoses, irrespective of the child's age, and that the assessment may not be fully in keeping with guidelines. Hopefully the UK National Health Service is doing better in this respect?

Visser, S. N., Zablotsky, B., Holbrook, J. R., et al (2015) Diagnostic experiences of children with attention-deficit/hyperactivity disorder. National Health Statistics Report, 3, 1-7.

\section{PTSD treatment breakthrough!?}

Dost-traumatic stress disorder (PTSD) is here to Pstay, as we humans, with our unique ability to create conflict among ourselves, ensure we have a constant supply of sufferers. Can we prevent its spread? Possibly, if we could work together, but we can't - Pandora's box remains open! Can we treat those affected? There are psychological and pharmacological methods and there is EMDR (eye movement desensitisation and reprocessing), all of which have shown some efficacy.

Dr Eugene Lipov, an anaesthesiologist and the medical director of the Global Post-traumatic Stress Injury Foundation in the USA, claims to have found a much more effective treatment. He has shown that blockade of the stellate ganglion, a method used for many years in the treatment of migraine and chronic pain, is also effective in 